\title{
Optimization of Active Muscle Force-Length Models Using Least Squares Curve Fitting
}

\author{
G. A. Mohammed and M. Hou
}

\begin{abstract}
The objective of this paper is to propose an asymmetric Gaussian function as an alternative to the existing active force-length models, and to optimize this model along with several other existing models by using the least squares curve fitting method. The minimal set of coefficients is identified for each of these models to facilitate the least squares curve fitting. Sarcomere simulated data and one set of rabbits extensor digitorum II experimental data are used to illustrate optimal curve fitting of the selected force-length functions. The results shows that all the curves fit reasonably well the simulated and experimental data, while the GordonHuxley-Julian model and asymmetric Gaussian function are better than other functions in terms of statistical test scores Root Mean Squared Error and R-squared. However the differences in RMSE scores are insignificant $(0.3 \sim 6 \%)$ for simulated data and $(0.2 \sim 5 \%)$ for experimental data. The proposed asymmetric Gaussian model and the method of parametrization of this and other force-length models mentioned above can be used in studies on active force-length relationships of skeletal muscles that generate forces to cause movements of human and animal bodies.
\end{abstract}

Index Terms-Muscle force-length relationship, least squares optimization, curve fitting, asymmetric Gaussian.

\section{INTRODUCTION}

A NALYTICAL models of muscles are important for comprehension and development of strategies for motor control and for clinical restoration of motion to paralyzed limbs through functional electrical stimulation [1]. The function of skeletal muscles is to contract and in so doing to apply a force against its environment [2]. The relationship between muscle lengths and generated forces can be described by numerous existing models. These models are ranged in formulations like cross-bridge to phenomenological models of muscle force output as a function of activation and muscle force-length-velocity relationship [3].

Gordon et al. [4] used frog muscle fibers to investigate the correlation between force and length in sarcomeres. Edman and Reggiani [5] stimulated isolated frog fibers to explore the sarcomere force-length relationship and fitted polynomial function to represent experimental data.

Manuscript received April 15, 2015; revised June 30, 2015; accepted August 05, 2015.

G. A. Mohammed is with School of Engineering, University of Hull, Hull, HU6 7RX, UK, E-mail: g.a.mohammed@2010.hull.ac.uk, on leave from the Faculty of Engineering, University of Koya, Kurdistan of Iraq.

M. Hou is with School of Engineering, University of Hull, Hull, HU6 7RX, UK, E-mail: m.hou@hull.ac.uk.

Color versions of one or more of the figures in this paper are available online at http://ieeexplore.iee.org.

Copyright (c) 2014 IEEE. Personal use of this material is permitted. However, permission to use this material for any other purposes must be obtained from the IEEE by sending an email to pubspermissions@ieee.org.
Siebert et al. [6] used nonlinear regression to determine the parameters of two different models of cat muscles and found the one from the two which best fits the experimental data. Also, Rassier [7] carried out a sequence of experiments on isolated sarcomeres and half sarcomeres of rabbit psoas muscles to investigate the mechanisms of residual force enhancement associated with the forcelength relationship, with a fitted fourth-order polynomial function representing the force-length relationship of sarcomeres. Bahler et al. [2] tested rat muscles to explore the dynamic properties of force-length-velocity relationship of the muscle. Moreover, Winters et al. [8] used muscles of rabbits to determine the isometric relationship between muscle active force and length by using Hill's two-element model consisting of an active contractile element and a passive elastic element connected in parallel.

To determine the active force-length relationship of muscle fibers, in experiments the active force is obtained by subtracting the predetermined passive force from the measured total force. Nevertheless, in order to study effects of muscle forces on movements, it is necessary to consider the force-length relationship of whole muscletendon complexes, for example, use of Hills three-element model. Rode et al. [9] showed that different connection schemes of the two elastic elements to the active element can have significant effects on the active cat soleus forcelength relationship.

In the literature, there are quite a few mathematical models for active muscle force-length relationship, such as the Gordon-Huxley-Julian (GHJ) diagram [4] which is a widely used piecewise linear model, for example in [6], [10], cubic splines interpolation of the critical points of the GHJ function [11], [12], sinusoidal wave [13], Bézier splines [14], fourth-order polynomial function [5], [7] and Gaussian function [13]-[15]. Besides, the Gaussian function is used in OpenSim, a software system for modeling and simulations of musculoskeletal structures, as part of Thelen's muscle model [16].

Although none of these muscle models, including the one to be introduced in this paper, has been derived from first principles of bio-mechanics and bio-chemistry, the GHJ model stands out as it was put forward by Gordon et al. [4] on the basis of the sliding filament theory, namely the cross-bridge hypothesis, whereby muscle force is considered to be produced through actin and myosin filaments sliding past each other. Interestingly, among others, Herzog et al. [17] showed that using only the lengths of the actin and myosin filaments in the GHJ model yields good estimations of measured sarcomere force-length relationship of cat skeletal muscles. It is no 
surprise that the GHJ model has gained wide acceptance over decades.

From an analytic point of view, a piecewise linear function such as the GHJ model includes four linear segments to represent the ascending limb, plateau and descending limb of the active force-length relationship. The cubic splines model normally requires five or more knots to form the active muscle force-length curve with functional continuity up to the second order derivatives. Bézier splines produce a curve that does not exceed the control points which are representative of the data of muscle force-length, and multiple Bézier curves are normally needed to fit a large amount of data. Polynomials are in a simple form and can fit the data reasonably well with a low order, while a fitted polynomial could have difficulties in covering the whole length range. The Gaussian model is a simple smooth function and widely used. However, the main drawback of the standard Gaussian function is that it cannot reflect the well-known asymmetry of the active skeletal muscle force-length relationship on both sides of optimal muscle length.

To fit a particular model with experimental data, the models normally needs to be parametrized. For example, piecewise linear functions, cubic splines and Bézier splines have control points which need to be represented by a minimal set of parameters, while the standard Gaussian and polynomial functions are already naturally parametrized. After parametrization, these different mathematical models can be optimized by using least squares optimization to fit the observed data. In this way, these models can be compared.

The objectives of this paper are the proposal of an asymmetric Gaussian function which is superior to the symmetric Gaussian function in terms of ability of fitting observed force-length data, parametrization and optimization of the most wisely used force-length functions by using the least squares method. Simulated data and a set of rabbits extensor digitorum II (EDII) data are used to illustrate the proposed Gaussian model along with optimization of various active force-length models.

\section{Methods}

It is well known that curve fitting is the most important application of the least squares method. Let $f(l, p)$ be a known function of $l$, parametrized in $p$ consisting of a minimal number of coefficients. The function is uniquely determined once the parameter set $p$ is known. Curve fitting is to find the optimal parameter set $p$ by minimizing the sum of squared differences between measured values $\bar{f}_{i}$ of $f$ and the values determined from the model $f\left(\bar{l}_{i}, p\right)$ for measured values $\bar{l}_{i}$ of $l$. That is, given $n$ measurement pairs $\left(\bar{l}_{i}, \bar{f}_{i}\right)$, finding out $p$ to minimize [18]

$$
s(p)=\sum_{i=1}^{n}\left(\bar{f}_{i}-f\left(\bar{l}_{i}, p\right)\right)^{2} .
$$

Several models of the active muscle force-length relationship in form of $f(l, p)$ are to be optimized with respect to the least squares criterion.

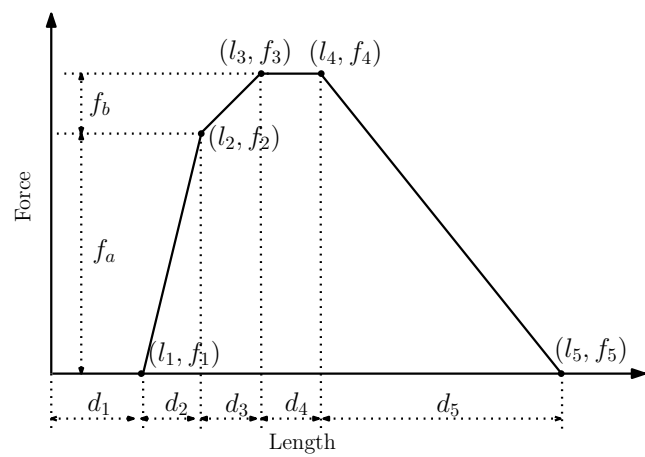

Fig. 1. Active force-length relationship

\section{A. Gordon-Huxley-Julian Diagram}

The isometric active force-length diagram of frog sarcomeres as elucidated by Gordon et al. [4] can be described by

$$
f(l)=a_{i}\left(l-l_{i}\right)+f_{i}, \quad l_{i} \leq l \leq l_{i+1}
$$

for $i=1,2,3,4$, where $f$ and $l$ are the force and length of the sarcomere, $a_{i}$ represents the slope of the linear function, and $\left(l_{i}, f_{i}\right)$ are the knots, as shown in Fig. 1. The five knots with ten coordinate coefficients determining the GHJ model can be specified with seven free positive parameters $\left(d_{1}, \cdots, d_{5}, f_{a}, f_{b}\right)$ as, for $i=1,2,3,4$,

$$
\begin{aligned}
& f_{1}=f_{5}=0, \quad f_{2}=f_{a}, \quad f_{3}=f_{4}=f_{a}+f_{b}, \\
& l_{1}=d_{1}, \quad l_{i+1}=l_{i}+d_{i+1}, \quad a_{i}=\frac{f_{i+1}-f_{i}}{d_{i+1}} .
\end{aligned}
$$

\section{B. Polynomial}

It is to fit just one fourth-order polynomial [5], [7], namely

$$
f(l)=b_{4} l^{4}+b_{3} l^{3}+b_{2} l^{2}+b_{1} l+b_{0}
$$

over the entire length range with the observed data. This is a smooth and very simple function, and no parametrization is needed.

\section{Cubic Splines}

The cubic spline model consists of piecewise third-order polynomials [11], [12]. Each of the polynomials passes two adjacent knots with continuous first and second order derivatives, and is in form of (3) but with $b_{4}=0$ over $l_{i} \leq l \leq l_{i+1}$ for $i=1, \cdots, 4$, where $l_{i}$ s are those defined in (2). Since the natural cubic spline is used [19], all the sixteen $(4 \times 4)$ parameters of the cubic spline model are uniquely determined by the five knots specified in the GHJ model and the requirements on the $C^{2}$-continuity of splines. This means that the cubic spline model is also parametrized by the seven coefficients of the GHJ model. 


\section{Bézier Curve}

A Bézier curve is a parametric curve considerably used in two-dimensional computer graphics to generate a smooth curve defined by a set of control points and it is $C^{2}$-continuous. A set of quintic Bézier curves have been used to represent active force-length relationship [14], but not in curve fitting context.

The five knots that specify the GHJ model are used in this paper with four extra points added into the middle of the five knots in order to have totally nine control points. These nine points are given by $p_{i}=\left[\begin{array}{c}\tilde{l}_{i} \\ \tilde{f}_{i}\end{array}\right]$, for $i=$ $1, \cdots, 9$, with

$$
\begin{aligned}
& {\left[\begin{array}{c}
\tilde{l}_{2 i-1} \\
\tilde{f}_{2 i-1}
\end{array}\right]=\left[\begin{array}{c}
l_{i} \\
f_{i}
\end{array}\right], \quad i=1, \cdots, 5} \\
& {\left[\begin{array}{c}
\tilde{l}_{2 i} \\
\tilde{f}_{2 i}
\end{array}\right]=\frac{1}{2}\left[\begin{array}{c}
\tilde{l}_{2 i-1}+\tilde{l}_{2 i+1} \\
\tilde{f}_{2 i-1}+\tilde{f}_{2 i+1}
\end{array}\right], \quad i=1, \ldots, 4}
\end{aligned}
$$

Five Bézier curves joint together are used to ensure better fit with respect to observed data, two of them are of degree one and the remaining three of degree two. The joint Bézier function is given by

$$
p(t)=\left[\begin{array}{c}
l(t) \\
f(t)
\end{array}\right], \quad 0 \leq t \leq 1
$$

with
a) in section $\left(\tilde{l}_{1}, \tilde{l}_{2}\right): p(t)=p_{1}+t\left(p_{2}-p_{1}\right)$;
b) in section $\left(\tilde{l}_{2 i}, \tilde{l}_{2(i+1)}\right): p(t)=(1-t)^{2} p_{2 i}+2(t-$ $\left.t^{2}\right) p_{2(i+1)}+t^{2} p_{2(i+1)}$, for $i=1,2,3$;
c) in section $\left(\tilde{l}_{8}, \tilde{l}_{9}\right): p(t)=p_{8}+t\left(p_{9}-p_{8}\right)$.

\section{E. Asymmetric Gaussian}

To accommodate the asymmetric nature of the active force-length relationship on both sides of the optimal length, an asymmetric Gaussian function is proposed. Parametrized by five coefficients $c_{0}, \cdots, c_{4}$ with $c_{0}, c_{1}$ and $c_{2}$ being positive, and $c_{3}$ and $c_{4}$ positive or negative, this function is given by

$$
f(l)=-c_{0}+c_{1} e^{-\left(\frac{l-c_{2}}{c_{3}+c_{4} l}\right)^{2}} .
$$

Coefficient $-c_{0}$ shifts the function down, $-c_{0}+c_{1}$ is the maximum isometric force, $c_{2}$ is the optimal length, and $\left(c_{3}+c_{4} l\right)^{2}$ is associated with the width of the function variable on both sides of the optimal length.

Fig. 2 is a plot of function (5) and defines a new set of coefficients $\left(l_{a}, l_{b}, l_{m}, f_{m}, r\right)$ parametrizing the function. Compared with the parameter set $\left(c_{0}, \cdots, c_{4}\right)$, the new parameters have clear physical meanings. Apparently, $l_{m}$ and $f_{m}$ are respectively the optimal length and force, $l_{a}$ and $l_{a}+l_{b}$ are respectively the minimal and maximal lengths, and $r$ is the slope of the curve at $l_{a}$. The mapping relations between these two sets of parameters are given in Appendix. It is usually convenient to specify initial values for $\left(l_{a}, l_{b}, l_{m}, f_{m}, r\right)$, and then through the inverse mapping, initial values of $\left(c_{0}, \cdots, c_{4}\right)$ can be obtained for least squares optimization.

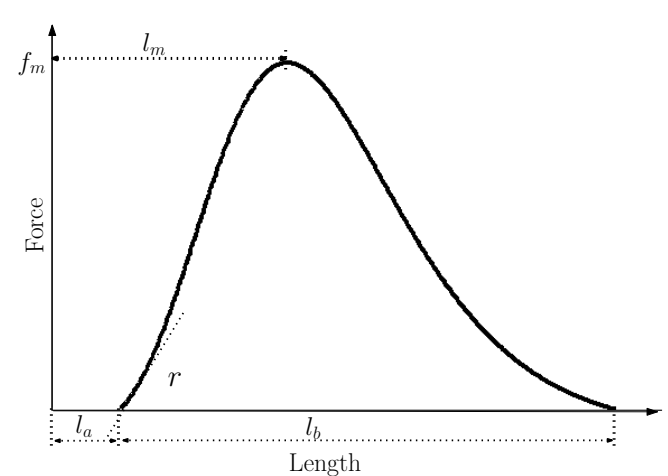

Fig. 2. Asymmetric Gaussian function

\section{F. Simulated Data}

To illustrate curve fitting of the force-length models described so far, simulated data were generated by using the original GHJ model for the whole range of length. Real experimental data of sarcomere force-length relationships would be ideal, nevertheless the data reported in the literature are usually sparse and do not cover the whole length range.

Since the GHJ diagram was determined from the experimental data on multiple muscle fibers over length segments, it is better understood as a representative model of multiple sarcomeres. This leads to a particular way of generating simulated data. In this study, the original GHJ model is used to to generate 10 other GHJ F-L functions through parameter variations by adding a sequence of noise to each of the seven parameters defining the GHJ model. Each of the ten individual GHJ functions was used to generate 20 simulated data by adding a sequence of noise to a pre-specified sequence of lengths, and another to the corresponding forces calculated from the GHJ mode. The sequences of noise are normally distributed with standard deviation $\sigma=0.13$ and mean $\mu=0$. In this way, 200 simulated data were generated in total. Furthermore, a second set of simulated data has been generated using the asymmetric Gaussian function defined in (5) and by repeating the same procedure described above.

The GHJ model was originally presented as tensionlength relationship. To be consistent with force measurements of the experimental data discussed later, tension $\left(\mathrm{km} / \mathrm{cm}^{2}\right)$ is converted to force $(\mathrm{N})$ by multiplying the tension by the gravitational acceleration $\left(9.81 \mathrm{~m} / \mathrm{s}^{2}\right)$ and the myofibril cross section area $\left(8.659 \times 10^{-7} \mathrm{~cm}^{2}\right)$ calculated from the frog myofibril mean diameter $(1.05 \mu \mathrm{m})$ in [20].

\section{G. Experimental Data}

The experimental data of the force-length relationship of rabbits extensor digitorum II (EDII) muscles [8] are used in curve fitting of the force-length models discussed in this paper. This set of data were collected from the experiments on the rabbits EDII muscles from 14 animal subjects. 


\section{H. Goodness Of Fit}

Goodness of fit measures how well the fitted mathematical model agrees with the observed data. Two usual criteria the root mean square error (RMSE) and R-square $\left(\mathrm{R}^{2}\right)$ are used for evaluation of fitness of the various force-length models with simulated and real data.

\section{Software Tool}

Matlab 2013b is used in the software coding for the curves fitted using least squares method. Also, the results have been checked directly by using the curve fitting toolbox cftool in Matlab.

\section{RESUlts AND Discussion}

The results for all five fitted functions (GHJ, asymmetric Gaussian, cubic splines, Bézier curve and polynomial) with the simulated data of sarcomeres force-length are shown in Fig. 3. As summarized in Table I, with the smallest RMSE and the biggest $\mathrm{R}^{2}$ scores, the GHJ function best fits the simulated data generated from the original GHJ model. The difference between the RMSEs of the fitted GHJ and asymmetric Gaussian models is only $(0.37 \%)$, while the difference between the RMSEs of the fitted GHJ and other models is around $(3 \sim 6 \%)$. However, it is not to say that there is a major difference among fitted functions. In general, any one of these fitted functions could be regarded as a good characterization of the sarcomeres force-length relationship with respect to the simulated data. The values of the scaled optimal force $f_{m}(\mathrm{~N})$ and optimal sarcomere length $l_{m}(\mu \mathrm{m})$, and the number of parameters used in a specific function are also included in Table I.

TABLE I

FITTED FUNCTIONS USING SIMULATED SARCOMERE DATA GENERATED FROM THE GHJ MODEL

\begin{tabular}{c|c|c|c|c|c}
\hline Fitted function & $\begin{array}{c}\text { RMSE } \\
\times 10^{-7}\end{array}$ & $\mathrm{R}^{2}$ & $\begin{array}{c}\text { Para. } \\
\text { No. }\end{array}$ & $\begin{array}{c}f_{m}(\mathrm{~N}) \\
\times 10^{-7}\end{array}$ & $\begin{array}{c}l_{m} \\
(\mu \mathrm{m})\end{array}$ \\
\hline GHJ Diagram & 0.270 & 0.854 & 7 & 2.25 & 2.13 \\
\hline Asym. Gaussian & 0.271 & 0.852 & 5 & 2.30 & 2.05 \\
\hline Cubic splines & 0.279 & 0.844 & 7 & 2.22 & 2.07 \\
\hline Bézier Curve & 0.278 & 0.846 & 7 & 2.27 & 2.15 \\
\hline Polynomial & 0.287 & 0.834 & 5 & 2.20 & 2.18 \\
\hline
\end{tabular}

To see effects of different simulated data sets on outcomes of curve fitting, the second set of simulated data generated from the asymmetric Gaussian model is used. Table II summarizes the outcomes of the fitting of the same previous five functions. It is noticeable that the asymmetric Gaussian function with the smallest RMSE and biggest $\mathrm{R}^{2}$ best fits the new simulated data compared with other fitted functions. This suggests that the least squares method is a reliable tool to pick up the best function fitting with simulated and experimental data.

The statistical test results of the fitted curves with EDII muscle experimental data [8] are shown in Fig. 4. As summarized in Table III, the GHJ function has the RMSE roughly similar to that of the asymmetric Gaussian function, and their RMSE values are slightly different from

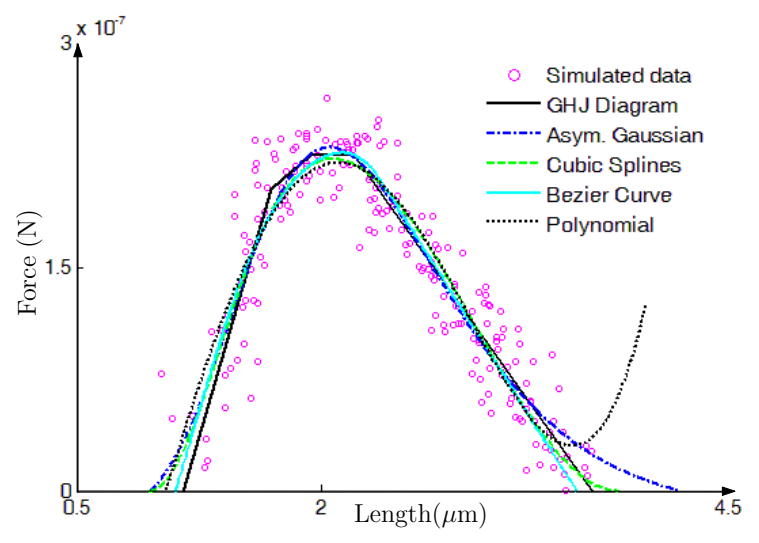

Fig. 3. Fitted functions with simulated sarcomere data generated from the GHJ model

TABLE II

FITTED FUNCTIONS USING SIMULATED SARCOMERE DATA GENERATED FROM THE ASYMMETRIC GAUSSIAN MODEL

\begin{tabular}{l|c|c}
\hline Fitted Functions & $\begin{array}{c}\text { RMSE } \\
\times 10^{-7}\end{array}$ & $\mathrm{R}^{2}$ \\
\hline GHJ Diagram & 0.440 & 0.727 \\
\hline Asym. Gaussian & 0.428 & 0.739 \\
\hline Cubic Splines & 0.452 & 0.712 \\
\hline Bezier Curve & 0.490 & 0.661 \\
\hline Polynomial & 0.462 & 0.695 \\
\hline
\end{tabular}

those of the cubic spline and Bézier function, whereas the polynomial function has the biggest RMSE value among the fitted curves. Again, the difference between the RMSEs of the fitted GHJ and asymmetric Gaussian models is very small $(0.2 \%)$, while the difference between the RMSEs of the fitted GHJ and other models is noticeable ( $2 \sim 5 \%)$. Furthermore, the polynomial function diverts away from its descending limb end shown in Fig. 4, which is consistent with the phenomena found in [7]. In general, the five fitted functions as shown in Fig. 4 have approximately the same goodness of fit and there is no significant difference between them in fitting with respect to observed data, except for the polynomial function. The values of the optimal force $f_{m}(\mathrm{~N})$ and optimal muscle length $l_{m}(\mathrm{~mm})$, and the number of parameters used in a specific function are also included in Table III.

TABLE III

FITTED FUNCTIONS WITH EDII MUSCLE DATA

\begin{tabular}{l|c|c|c|c|c}
\hline Fitted & RMSE & $\mathrm{R}^{2}$ & $\begin{array}{c}\text { Para. } \\
\text { No. }\end{array}$ & $\begin{array}{c}f_{m} \\
(\mathrm{~N})\end{array}$ & $\begin{array}{c}l_{m} \\
(\mathrm{~mm})\end{array}$ \\
\hline GHJ Diagram & 2.698 & 0.841 & 7 & 19.75 & 56.77 \\
\hline Asym. Gaussian & 2.704 & 0.840 & 5 & 20.23 & 56.32 \\
\hline Cubic Splines & 2.749 & 0.835 & 7 & 19.76 & 56.41 \\
\hline Bézier Curve & 2.745 & 0.836 & 7 & 19.48 & 56.68 \\
\hline Polynomial & 2.821 & 0.824 & 5 & 19.54 & 56.67 \\
\hline
\end{tabular}

Other aspects of comparing these fitted functions include examination of the degrees of their smoothness, complexity of computer coding and the number of parameters used. First, the GHJ function is $C^{0}$ continuous, cubic spline and Bézier are $C^{2}$-continuous whilst the asymmetric Gaussian and the polynomial functions are 


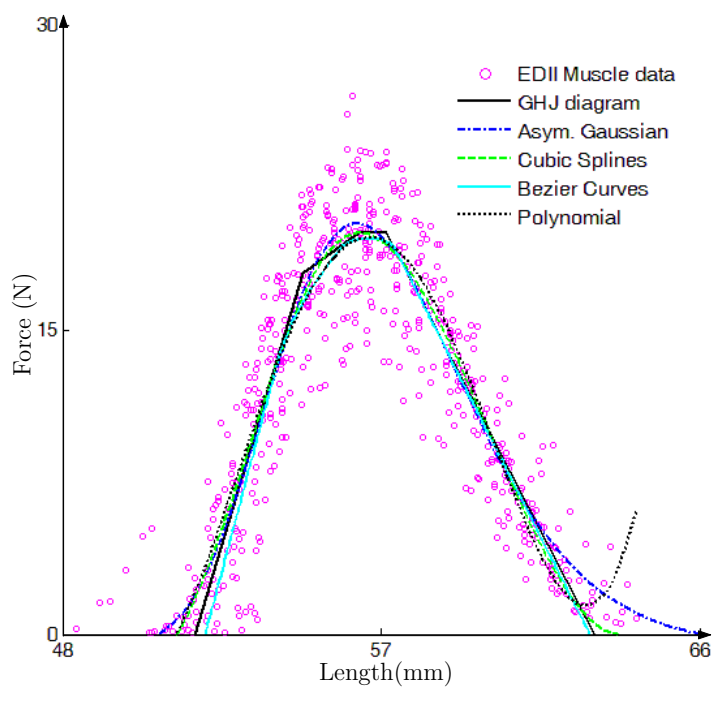

Fig. 4. Fitted functions with EDII muscle data

smooth functions with $C^{\infty}$-continuity. Second, the asymmetric Gaussian and polynomimal are a single function compared with the multiple segmental functions of GHJ, cubic splines and Bézier curves. The polynomial is the simplest function without need of parametrization. Third, the asymmetric Gaussian and polynomial functions have five free parameters whereas the other three functions need seven parameters. Additionally, the optimal isometric force $f_{m}$ and optimal length $l_{m}$ can be automatically found from the fitted curves and or from the optimal parameters. Note that the GHJ model has been normalized by the optimal force and length and hence parametrized with five parameters and fitted using non-linear regression [6]. Experimental data are naturally not normalized. Although normalization of the data by $f_{m}$ and $l_{m}$ would reduce the number of free coefficients parametrizing a force-length function as often done in the published reports, it is not recommended for curve fitting because true $f_{m}$ and $l_{m}$ are never precisely known. Normalization of a particular set of data based on approximate values of $f_{m}$ and $l_{m}$ before curve fitting will normally reduce fitness of the fitted functions with respect to the whole set of data. Nevertheless, if needed, it is straightforward to normalize a fitted function $y=f(l, p)$ simply as $\bar{y}=f\left(l_{m} \bar{l}, p\right) / f_{m}$, where $\bar{y}$ and $\bar{l}$ are respectively the normalized force and length.

The asymmetric Gaussian function appears to be new as it has not been found in the literature across scientific disciplines. In probability theory and statistics, the skew normal distribution [21] is an asymmetric function, built as a product of the normal distribution function and its cumulative distribution function. The skew normal function is hence complicated than the asymmetric Gaussian function, and more importantly the former has too few parameters to fit muscle force-length data. Another modified Gaussian function in probability theory and statistics is the split normal distribution [22] which combines two normal distributions with different standard deviations on both sides of the center of the distribution. Although the split normal function could fit well muscle force-length data, it is a two-segmental function and no longer smooth at the junction of the two half normal functions.

Finally, it has been seen that the 4th-order polynomial shows divergence towards the further end of the length range. Because of that an increase of the polynomial order would not be considered appropriate. However, by introducing an extra parameter in the asymmetric Gaussian function, the modified Gaussian model is able to outperform the GHJ model. For example, fitting of the modified asymmetric Gaussian function

$$
f(l)=-c_{0}+c_{1} e^{-\left(\frac{l-c_{2}}{c_{3}+c_{4} l+\alpha(l)}\right)^{2}}
$$

with $\alpha(l)=c_{5} l^{2}$ or $\alpha(l)=c_{5} / l$, is able to reduce the RMSE from 2.692 to 2.654 which is less than all others RMSE of fitted curves using EDII experiment data. A similar effect was also observed when fitting this modified asymmetrical Gaussian with the simulated forcelength data, where the RMSE is reduced from $0.271 \times 10^{-7}$ to $0.264 \times 10^{-7}$. However, the suggested formats have an extra parameter and do not appear as simple as the originally proposed asymmetric Gaussian function.

\section{CONCLUSION}

The asymmetric Gaussian proposed in this paper is a simple and smooth function for active muscle force-length relationships. This function appears to be novel and may also have potential in other applications.

By using the least squares method, the asymmetric Gaussian model along with the well-known GordonHuxley-Julian diagram and other models such as cubic splines, Bézier curve and polynomial have been optimized and compared with respect to fitting of simulated data and one set of experimental data. The results show that the Gordon-Huxley-Julian diagram and asymmetric Gaussian best fit the simulated and experimental data in comparison with other models studied in this paper. The differences between these models in terms of root mean square errors are however insignificant $(0.2 \sim 5 \%)$ for experimental EDII data and $(0.3 \sim 7 \%)$ for simulated data.

Only five coefficients parametrize the asymmetric Gaussian, while other models (except for the fourth-order polynomial) considered in this paper require seven coefficients. By including an extra parameter, but at the expense of having slightly increased complex, the asymmetric Gaussian model can clearly outperform all other models considered in this study.

\section{APPENDIX}

\section{A. Parameter mapping}

The forward mapping of the asymmetric Gaussian function from parameter set $\left(c_{0}, \cdots, c_{4}\right)$ to $\left(l_{a}, l_{b}, f_{m}, l_{m}, r\right)$ is

$$
\begin{aligned}
l_{m} & =c_{2}, \quad f_{m}=-c_{0}+c_{1}, \\
l_{a} & =\frac{c_{2}-a c_{3}}{1+a c_{4}}, \quad l_{b}=-l_{a}+\frac{c_{2}+a c_{3}}{1-a c_{4}} \\
r & =-2 c_{0}\left(c_{3}+c_{2} c_{4}\right) \frac{l_{a}-c_{2}}{\left(c_{3}+c_{4} l_{a}\right)^{3}}
\end{aligned}
$$


with $a=\sqrt{\ln \frac{c_{1}}{c_{0}}}$. Note that in all cases of fitting forcelength data, $c_{1}$ is greater than $c_{0}$. This ensures that $a$ is a well defined real number.

The inverse mapping is given by

$$
\begin{aligned}
r & =\frac{4 c_{0}\left(l_{a}+l_{b}-l_{m}\right)}{l_{b}\left(l_{m}-l_{a}\right)} \ln \left(\frac{f_{m}+c_{0}}{c_{0}}\right), \\
c_{1} & =f_{m}+c_{0}, \quad c_{2}=l_{m}, \\
c_{3} & =\frac{l_{b}\left(l_{m}-l_{a}\right)}{a l_{b}}-l_{a} c_{4}, \quad c_{4}=\frac{2 l_{a}+l_{b}-2 l_{m}}{a l_{b}},
\end{aligned}
$$

where $c_{0}$ needs to be numerically solved from the first equation which is implicit in $c_{0}$, and the remaining $c_{i}$ s are then readily obtained.

\section{B. The optimal parameters of fitted functions with simu- lated data}

1) GHJ diagram: $d_{1}=1.157, d_{2}=0.525, d_{3}=0.266$, $d_{4}=0.276, d_{5}=1.420, f_{a}=1.949 \times 10^{-7}, f_{b}=0.296 \times$ $10^{-7}$.

2) Cubic splines: $d_{1}=0.997, d_{2}=0.262, d_{3}=0.171$, $d_{4}=1.442, d_{5}=0.928, f_{a}=0.584 \times 10^{-7}, f_{b}=0.644 \times$ $10^{-7}$.

3) Bézier curve: $d_{1}=1.108, d_{2}=0.510, d_{3}=0.400$, $d_{4}=0.247, d_{5}=1.308, f_{a}=1.927 \times 10^{-7}, f_{b}=0.339 \times$ $10^{-7}$.

4) Asymmetric Gaussian: $c_{0}=0.144 \times 10^{-7}, c_{1}=$ $2.437 e \times 10^{-7}, c_{2}=2.041, c_{3}=0.474, c_{4}=0.200$ $\left(l_{a}=0.953, l_{b}=3.254, l_{m}=2.052, f_{m}=2.300 \times 10^{-7}\right.$, $\left.r=1.091 \times 10^{-7}\right)$.

5) Polynomial: $b_{4}=0.345 \times 10^{-7}$,

$b_{3}=-2.617 \times 10^{-7}, b_{2}=5.308 \times 10^{-7}$,

$b_{1}=-0.405 \times 10^{-7}, b_{0}=-2.824 \times 10^{-7}$.

\section{The optimal parameters of fitted functions with EDII muscle empirical data}

1) GHJ diagram: $d_{1}=51.748, d_{2}=3.027, d_{3}=$ $1.601, d_{4}=0.782, d_{5}=5.872, f_{a}=17.788, f_{b}=1.958$.

2) Cubic splines: $d_{1}=51.226, d_{2}=2.891, d_{3}=$ $1.332, d_{4}=1.998, d_{5}=6.292, f_{a}=13.640, f_{b}=5.061$.

3) Bézier curve: $d_{1}=52.026, d_{2}=2.438, d_{3}=1.525$, $d_{4}=1.161, d_{5}=5.672, f_{a}=16.54, f_{b}=3.348$.

4) Asymmetric Gaussian: $c_{0}=0.712, c_{1}=20.940$, $c_{2}=56.323, c_{3}=-4.336, c_{4}=0.146\left(l_{a}=50.715\right.$, $\left.l_{b}=15.321, l_{m}=56.323, f_{m}=20.228, r=1.088\right)$.

5) Polynomial: $b_{4}=0.012, b_{3}=-2.788, b_{2}=235.4$, $b_{1}=-8795, b_{0}=1.227 e+5$.

\section{ACKNOWLEDGMENTS}

The authors are grateful to Samuel R. Ward* for making the rabbit muscles experimental data available to this study.

*Department of Radiology, University of California and Veterans Administration Medical Centers, 3350 La Jolla Village Drive, San Diego, California 92161, USA

\section{REFERENCES}

[1] I. E. Brown and G. E. Loeb, "Measured and modeled properties of mammalian skeletal muscle: Iv. dynamics of activation and deactivation," Journal of Muscle Research \& Cell Motility, vol. 21, no. 1, pp. 33-47, 2000.

[2] A. S. Bahler, J. T. Fales, and K. L. Zierler, "The dynamic properties of mammalian skeletal muscle," The Journal of general physiology, vol. 51, no. 3, pp. 369-384, 1968.

[3] R. L. Lieber, C. G. Brown, and C. L. Trestik, "Model of muscletendon interaction during frog semitendinosis fixed-end contractions," Journal of biomechanics, vol. 25, no. 4, pp. 421-428, 1992.

[4] A. Gordon, A. F. Huxley, and F. Julian, "The variation in isometric tension with sarcomere length in vertebrate muscle fibres," The Journal of physiology, vol. 184, no. 1, pp. 170-192, 1966.

[5] K. Edman and C. Reggiani, "The sarcomere length-tension relation determined in short segments of intact muscle fibres of the frog." The Journal of physiology, vol. 385, no. 1, pp. 709-732, 1987.

[6] T. Siebert, C. Rode, W. Herzog, O. Till, and R. Blickhan, "Nonlinearities make a difference: comparison of two common hill-type models with real muscle,' Biological cybernetics, vol. 98, no. 2, pp. 133-143, 2008.

[7] D. E. Rassier, "Residual force enhancement in skeletal muscles: one sarcomere after the other," Journal of muscle research and cell motility, vol. 33, no. 3-4, pp. 155-165, 2012.

[8] T. M. Winters, M. Takahashi, R. L. Lieber, and S. R. Ward, "Whole muscle length-tension relationships are accurately modeled as scaled sarcomeres in rabbit hindlimb muscles," Journal of biomechanics, vol. 44, no. 1, pp. 109-115, 2011.

[9] C. Rode, T. Siebert, W. Herzog, and R. Blickhan, "The effects of parallel and series elastic components on the active cat soleus force-length relationship," Journal of Mechanics in Medicine and Biology, vol. 9, no. 01, pp. 105-122, 2009.

[10] R. Maas, T. Siebert, and S. Leyendecker, "On the relevance of structure preservation to simulations of muscle actuated movements," Biomechanics and modeling in mechanobiology, vol. 11, no. 3-4, pp. 543-556, 2012.

[11] D. G. Lloyd and T. F. Besier, "An emg-driven musculoskeletal model to estimate muscle forces and knee joint moments in vivo," Journal of biomechanics, vol. 36, no. 6, pp. 765-776, 2003.

[12] Y. DeWoody, C. Martin, and L. Schovanec, "A forward dynamic model of gait with application to stress analysis of bone," Mathematical and computer modelling, vol. 33, no. 1, pp. 121-143, 2001.

[13] I. E. Brown, S. H. Scott, and G. E. Loeb, "Mechanics of feline soleus: Ii design and validation of a mathematical model," Journal of Muscle Research \& Cell Motility, vol. 17, no. 2, pp. 221-233, 1996.

[14] M. Millard, T. Uchida, A. Seth, and S. L. Delp, "Flexing computational muscle: modeling and simulation of musculotendon dynamics," Journal of biomechanical engineering, vol. 135, no. 2, p. $021005,2013$.

[15] J. M. Winters, "An improved muscle-reflex actuator for use in large-scale neuromusculoskeletal models," Annals of biomedical engineering, vol. 23, no. 4, pp. 359-374, 1995.

[16] D. G. Thelen, "Adjustment of muscle mechanics model parameters to simulate dynamic contractions in older adults," Journal of biomechanical engineering, vol. 125, no. 1, pp. 70-77, 2003.

[17] W. Herzog, S. Kamal, and H. Clarke, "Myofilament lengths of cat skeletal muscle: theoretical considerations and functional implications," Journal of biomechanics, vol. 25, no. 8, pp. 945-948, 1992.

[18] H. Motulsky and A. Christopoulos, Fitting models to biological data using linear and nonlinear regression: a practical guide to curve fitting. Oxford University Press, 2004.

[19] W. H. Press, Numerical recipes 3rd edition: The art of scientific computing. Cambridge university press, 2007.

[20] B. A. Mobley and B. R. Eisenberg, "Sizes of components in frog skeletal muscle measured by methods of stereology." The Journal of general physiology, vol. 66, no. 1, pp. 31-45, 1975.

[21] W. L. Martinez and A. R. Martinez, Computational Statistics Handbook with MATLAB. Chapman and Hall/CRC, 2002.

[22] K. F. Wallis et al., "The two-piece normal, binormal, or double gaussian distribution: its origin and rediscoveries," Statistical Science, vol. 29, no. 1, pp. 106-112, 2014. 